\title{
Use of horizontal ultrathin gel electrophoresis to analyze allelic deletions in chromosome band 11 p15.5 in gliomas ${ }^{1}$
}

\author{
Irene F. Newsham, Karen M. Gorse, Sandra A. Rempel, John Luckey, James B. Golden, \\ and Oliver Bögler ${ }^{2}$
}

\author{
Departments of Anatomy (I.F.N., K.M.G., O.B.), Pathology (I.F.N.), and Neurosurgery (O.B.), Medical College \\ of Virginia Campus at Virginia Commonwealth University, Richmond, VA 23298; Department of Neurosurgery, \\ Henry Ford Health Sciences Center, Detroit, MI 48202 (S.A.R.); and GeneSys Technologies, Inc., 1100 Dallas \\ Street, Sauk City, WI 53583 (J.L., J.B.G.)
}

\begin{abstract}
The prognosis for most patients with astrocytic glioma is poor, and postoperative life expectancy has not significantly improved in the last decade despite advances in diagnosis, surgery, and adjuvant therapy. Progress has been made, however, in cataloging the genetic alterations that occur in these tumors. Studying the allelic changes using loss of heterozygosity analysis has proven to be a reliable and rapid way of identifying genetic alterations fundamental to the pathology of this disease. In this study, we used a series of fluorescent-labeled markers and a new horizontal ultrathin gel electrophoresis technology (HUGE; GeneSys Technologies, Inc.) to analyze loss of heterozygosity on $11 \mathrm{p} 15$ in a series of 24 matched nor$\mathrm{mal} /$ tumor glioma pairs that included both anaplastic astrocytomas and glioblastomas. These studies significantly narrowed the region harboring a putative $11 \mathrm{p} 15.5$ glioma-associated gene and further suggest that a second gene involved in the pathogenesis of brain tumors may exist, centromeric, in bands 11p15.5-p15.4. Neuro-Oncology 2, 1-5, 2000 (Posted to Neuro-Oncology [serial online], Doc. 99-14, December 10, 1999. URL <neurooncology.mc.duke.edu>)
\end{abstract}

Received 1 April 1999, accepted 2 July 1999.

${ }^{1}$ This work was funded in part by grants from the Rainbow Foundation (O.B.) and the Hord Foundation (O.B.).

\footnotetext{
${ }^{2}$ Address correspondence and reprint requests to Oliver Bögler, Ph.D., Departments of Anatomy and Neurosurgery, Virginia Commonwealth University, PO Box 980709, Richmond, VA 23298-0709.
}

\footnotetext{
${ }^{3}$ Abbreviations used are as follows: $\mathrm{CM}$, centiMorgan; GBM, glioblastoma multiforme; HUGE, horizontal ultrathin gel electrophoresis; $\mathrm{LOH}$, loss of heterozygosity.
}

The prognosis for most patients with astrocytic glioma is dismal. Postoperative survival of a patient with $\mathrm{GBM}^{3}$, the most aggressive astrocytoma, is often less than one year. Life expectancy has not significantly improved in the last decade despite advances in diagnosis, surgery, and adjuvant therapy. Furthermore, traditional diagnostics by histology have not improved beyond the occasional inclusion of some immunohistochemical markers such as glial fibrillary acidic protein. In contrast, significant progress has been made in cataloging genetic alterations that occur in brain tumors. Allelic deletions of several chromosomal loci have been described (Furnari et al., 1995). Studying these allelic changes using $\mathrm{LOH}$ analysis is a reliable and rapid way of identifying genetic alterations fundamental to the pathology of this disease. For some of these regions in brain tumors, the critical gene has been identified, such as the PTEN/MMAC1 gene on chromosome 10 (Li et al., 1997; Steck et al., 1997). However, when a gene at the center of the region of genetic loss is not yet characterized, $\mathrm{LOH}$ can itself serve as a marker of tumor progression.

Analysis of the incidence of these various genetic alterations in gliomas has revealed that they are associated with different histologic subtypes (e.g., LOH on 1p, which is common in oligodendrocytic gliomas; Bello et al., 1994; Kraus et al., 1995) and different grades within a given subtype. In pathways of progression, it has even been possible to classify some mutations as early and others as late in terms of when they occur (Furnari et al., 1995). For example, mutation and allelic deletion of the TP53 gene occurs early and is found in about $35 \%$ to $40 \%$ of astrocytic gliomas (Bögler et al., 1995). In addition to giving an indication of the tumor stage, there is evidence that some genetic alterations may be excellent 
prognostic indicators. For example, de novo GBMs are more aggressive tumors with a corresponding poor prognosis. A series of studies have shown that this form of GBM tumor does not show LOH for chromosome $17 \mathrm{p}$ or concomitant TP53 mutation. However, these mutations occur frequently in the progressive form of GBM (von Deimling et al., 1992).

A recent molecular study localized a gene involved in malignant gliomas to a broad area distal to D11S988 on chromosome $11 \mathrm{p} 15$, a region encompassing much of the $11 \mathrm{p} 15.5$ band (Sonoda et al., 1995). Using a series of fluorescent-labeled markers, including three distal to D11S988, and new electrophoresis technology called HUGE (GeneSys Technologies, Inc., Sauk City, WI), we analyzed LOH on 11 p 15 in a series of 24 matched nor$\mathrm{mal} /$ tumor glioma pairs including both anaplastic astrocytomas and glioblastomas, with the goal of identifying a refined minimal region of allelic deletion containing this putative chromosome 11 tumor suppressor gene. These studies significantly narrowed the region harboring this $11 \mathrm{p} 15.5$ glioma-associated gene and further suggest that a second gene involved in the pathogenesis of brain tumors may exist, centromeric, in bands $11 \mathrm{p} 15.5-\mathrm{p} 15.4$.

\section{Materials and Methods}

\section{Patient Tissue Samples}

Glioma specimens were divided immediately upon surgical removal. Partial tumor specimens were fixed in $10 \%$ buffered formalin and embedded in paraffin for pathological diagnosis. The remaining tumor tissue was immediately snap-frozen in liquid nitrogen and later processed for DNA as previously reported (Tran et al., 1998). Blood specimens were collected at the time of surgery and stored at $4^{\circ} \mathrm{C}$. Peripheral blood lymphocyte DNA was isolated within three days using a DNA extraction kit according to manufacturer's instructions (Stratagene, La Jolla, CA). Informed consent on a form approved by the Institutional Review Board was obtained from all patients or their guardians. Pathology reports were reviewed and specimens were selected at random on the basis of availability of both normal and tumor DNA.

\section{Microsatellite Polymorphism and Amplification}

LOH for chromosomal band $11 \mathrm{p} 15.5$ was examined using six CA-repeat-based polymorphic markers: telomeric to centromeric, D11S2071-D11S1363-D11S922D11S988-D11S1338-D11S909. The forward primers for each of these markers were synthesized (GeneSys Technologies, Inc.) with either a 6-carboxyfluorescein (FAM) or 4,7,2',7'-tetrachloro-6-carboxyfluorescein (TET) (Applied BioSystems, Inc., Foster City, CA) fluorescentdye tag. Amplification was performed on $25 \mathrm{ng}$ of genomic DNA from tumor tissue and peripheral blood lymphocyte DNA in 25- $\mu$ l reactions. The polymerase chain reaction protocols used for these markers have been described previously (Tran and Newsham, 1996). Amplification products were precipitated and resuspended in 5 $\mu$ of water.

\section{HUGE}

GeneSys Technologies' electrophoretic (HUGE) highthroughput fluorescence-based DNA fragment analyzer (GTI-9600) was applied to the separation and analysis alleles generated by polymerase chain reaction. For loading, $1 \mu \mathrm{l}$ of sample from polymerase chain reaction was added to $2 \mu \mathrm{l}$ of formamide loading dye (Amersham, Inc., Piscataway, NJ). For size estimation, $0.8 \mu \mathrm{l}$ of RO3350 fluorescent size markers (Applied BioSystems, Inc.) was added to tumor DNA amplification products. Samples were heated to $95^{\circ} \mathrm{C}$, placed on ice, and loaded on a $75-\mu \mathrm{m}, 6 \%$ polyacrylamide gel in the GTI-9600 and run for $2 \mathrm{~h}$ at $30 \mathrm{~W}$ in $1 \mathrm{X}$ Tris borate EDTA (TBE). While the samples were running, data were collected and stored using the accompanying Cartographer data analysis software program (GeneSys Technologies, Inc.). Cartographer readily extracts the pixel intensity of individual peaks present in a lane trace, allowing for sizing of alleles and quantitation of fluorescent peak areas, i.e., area under the curve. In determining whether allelic deletion had occurred at individual markers, we calculated allelic ratios and expressed them as a percentage of loss of intensity for the tumor alleles compared with the corresponding normal allele ( $\mathrm{D}$ value). $\mathrm{LOH}$ is assessed by alterations in the allelic ratio observed at a given locus between normal tissue and tumor tissue. It does not require a 1:1 allelic ratio in the normal tissue. Although $100 \%$ loss of one allele would be expected in a pure sample of a clonal tumor that had undergone LOH (Cawkwell, et al., 1993), this is rarely observed in tumor biopsy samples. This is because such samples also contain normal cells, and also because tumors can be heterogeneous with respect to genetic loss, particularly those that occur later in the progression of such tumors. In order to allow for this, LOH was considered present when the calculated loss ratio for a tumor allele was $>40 \%$ (D0.40). A loss ratio between $30 \%$ and $40 \%$ (D0.30-D0.40) was termed allelic imbalance in order to indicate the possibility that $\mathrm{LOH}$ was occurring at this locus but was not likely to be present in most of the sample.

\section{Results}

Matched pairs of normal and brain tumor DNA from 24 malignant gliomas were assessed for allelic deletions at 6 loci in 11p15.4-pter using HUGE analysis. This set of tumors, categorized according to the WHO grading system (Kleihues et al., 1993), included 2 astrocytomas, 7 anaplastic astrocytomas, and 15 glioblastomas. The overall frequency of $\mathrm{LOH}$ on $11 \mathrm{p} 15$ in these samples was $33 \%$ (8 of 24 ), a value consistent with that previously reported for malignant gliomas $(26 \%-35 \%$; Sonada et al., 1995; Fults et al., 1992). Examples of HUGE allelic scans for tumor samples 491 and 316 are shown in Fig. 1. These tumors represent samples for which the presence or absence of $\mathrm{LOH}$ was difficult to assess by eye visualization of allelic scans alone. With the accompanying HUGE analytical software (Cartographer), precise and sensitive calculation of peak areas makes genotypic 

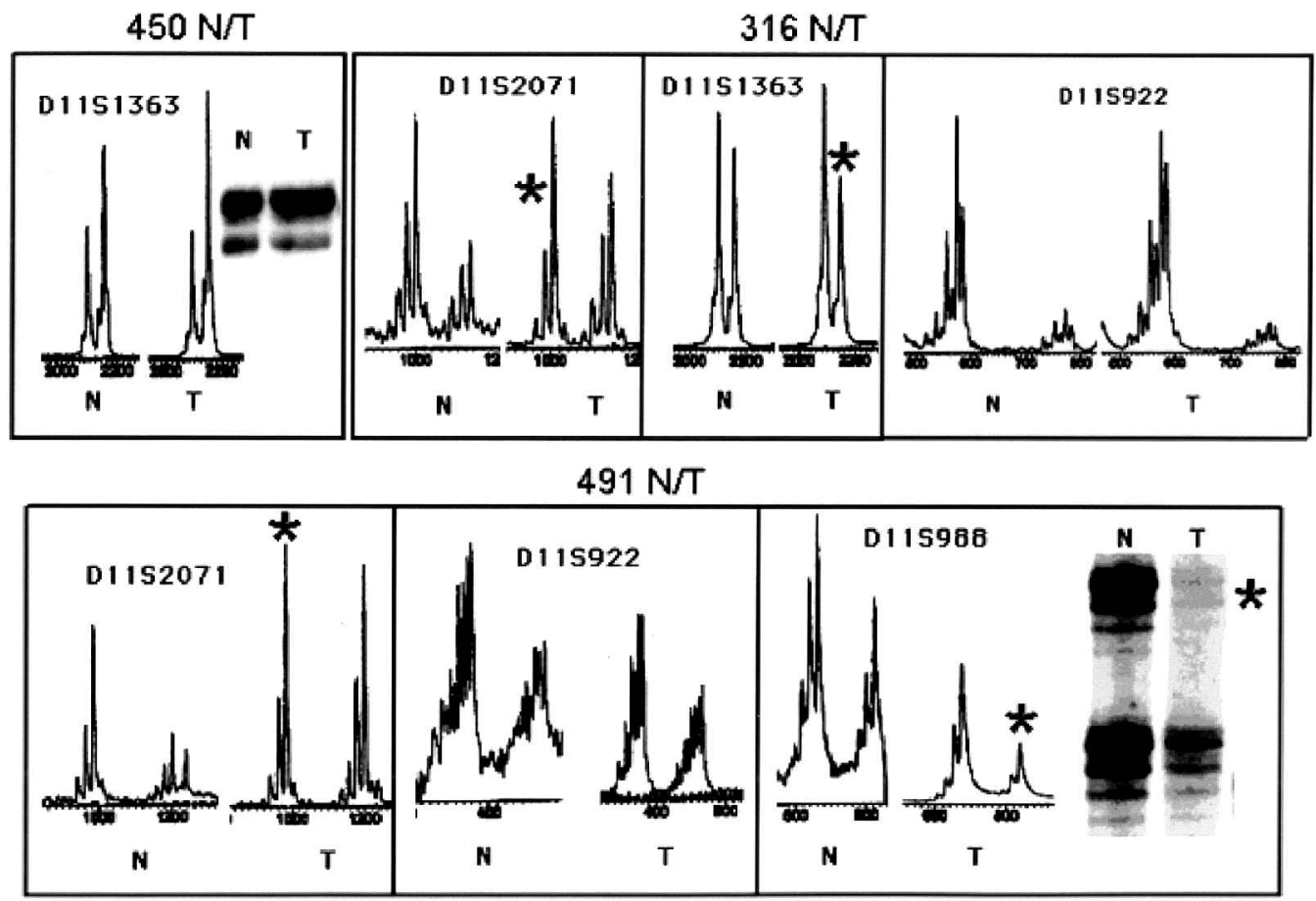

Fig. 1. Examples of allelic scans for chromosome 11p15.5 markers. Scans represent normal $(N)$ and matched tumor (T) DNA samples from the patients whose case numbers are shown above. Case $316 \mathrm{~N} / \mathrm{T}$ represents a glioblastoma multiforme (GBM) and cases $450 \mathrm{~N} / \mathrm{T}$ and $491 \mathrm{~N} / \mathrm{T}$ are anaplastic astrocytomas. Chromosome 11p15.5 loci names are shown at the top of scan boxes. Asterisks depict the densitometrically evaluated allelic reduction in the intensity of alleles between tumor and normal DNA samples. Please note that relative peak heights are used to determine loss of heterozygosity (LOH). Conventional autoradiographic LOH profiles of markers D11S1363 (Case 450) and D11S988 (Case 491 N/T) are included for comparison.

analysis with the GTI-9600 straightforward and accurate. In tumor sample 491, $\mathrm{LOH}$ was detected at distal marker D11S2071 (D0.57), but not at more proximal D11S922 (D0.17) (Fig. 1). A potential second region of $\mathrm{LOH}$ in tumor 491 was identified with $\mathrm{LOH}$ at proximal markers D11S988 and D11S909, extending into chromosomal band 11p15.4 (Fig. 2). The extent to which this separate region of $\mathrm{LOH}$ involves additional chromosome 11q sequences was not investigated further. For tumor 316, LOH/allelic imbalance was detected at $11 \mathrm{p} 15.5$ distal to D11S922 and secondarily proximal to D11S1338. Only two of the eight tumors exhibiting $\mathrm{LOH}$ did so at all markers analyzed, suggesting that loss of the entire chromosome 11 is not a common mutational mechanism in gliomas. Also, such whole chromosomal loss was not found to be predominant in breast and lung cancer (Tran and Newsham, 1996).

Comparative analysis of $\mathrm{LOH}$ for tumor samples 142, 316,223 , and 491 reveals a shared 3-cM region of allelic deletion between telomeric marker D11S2071 and centromeric marker D11S922 (Fig. 2). This distal LOH region overlaps with but represents a substantial refinement from the $21-\mathrm{cM}$ region described previously (Sonada et al., 1995). Individual marker deletion frequencies in this study peaked at marker D111363 (28\%), which is located in this smallest region of overlap, suggesting D11S1363 most closely approximates the location of a putative brain tumor suppressor gene. The $\mathrm{LOH}$ results for tumor 450 could potentially allow for further refine- ment of the $\mathrm{LOH}$ region to one bordered proximally by D11S1363. However, the allelic ratio at D11S1363 was only calculated to be D 0.29 , barely below the D 0.30 value established for allelic imbalance. Therefore we chose not to use this sample for $\mathrm{LOH}$ region refinement. Additional samples indicating marker D11S1363 as the proximal boundary are necessary to confidently refine the $11 \mathrm{p} 15.5 \mathrm{LOH}$ region to the D11S2071-D11S1363 region.

Chromosome 11p15.5 LOH was previously found to be specific for more advanced gliomas (Sonada et al., 1995). In this tumor set, $\mathrm{LOH}$ frequencies of $0 \%$ (0 of 2 ) for astrocytomas, $50 \%$ (4 of 8 ) for anaplastic astrocytomas, and $27 \%$ (4 of 15 ) for glioblastomas were seen, supporting the notion that the $11 \mathrm{p} 15.5$ gene or genes affected are more likely involved in the malignant progression of brain tumors. However, the number of samples representative of these lower grades must be expanded before statistically significant conclusions can be drawn.

\section{Discussion}

Clustering of high-frequency LOH for the short arm of chromosome 11 , specifically $11 \mathrm{p} 15.5$, occurs in a broad spectrum of adult tumors including bladder carcinomas (Shaw et al., 1995), adenocarcinomas of the stomach (Baffa et al., 1996), breast carcinomas (Karnik et al., 


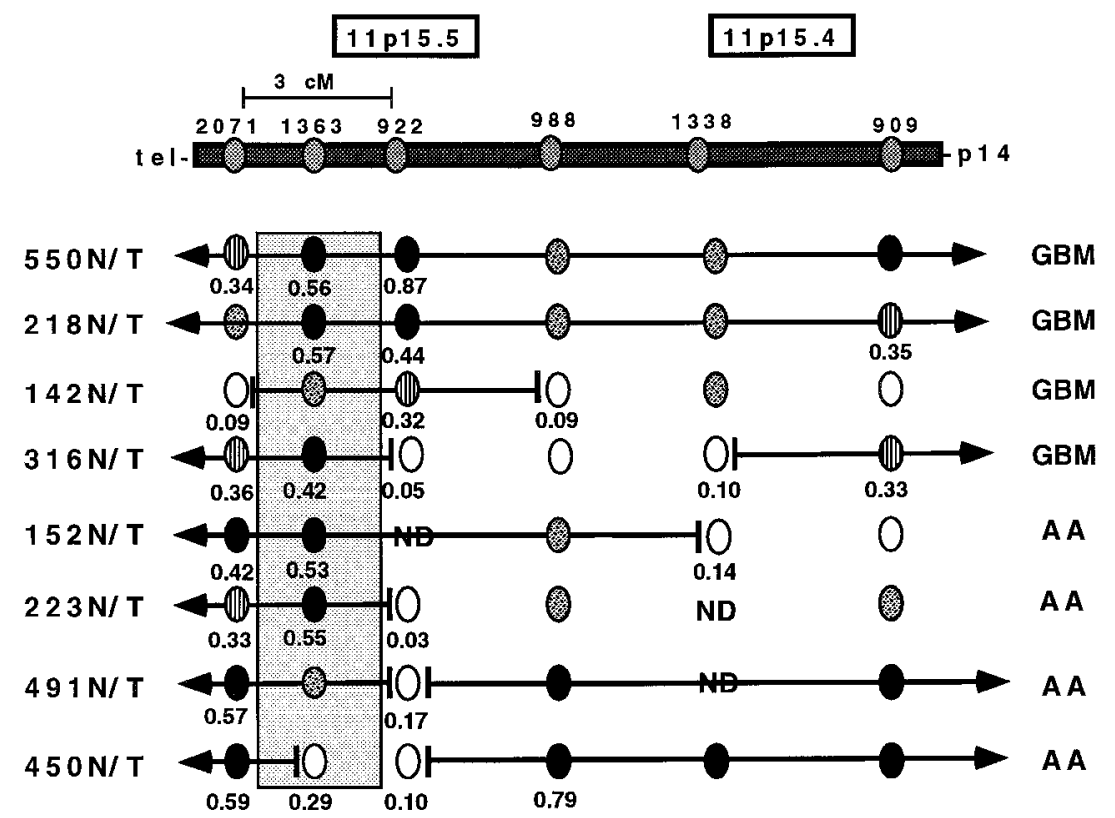

Fig. 2. Schematic representation of the regions of allelic deletion measured in eight gliomas. The 11p15.5 loci analyzed are shown telomeric (left) to centromeric (right) along the top. Numbers to the left are tumor case numbers. The shaded area indicates the smallest shared region of LOH defined in this analysis. Calculated D values for $\mathrm{LOH}$ appear beneath markers critical for defining allelic deletion regions. $\bullet, \mathrm{LOH} ; \bigcirc$, maintenance of heterozygosity; $\square$, not informative; $\mathbb{l l}$, allelic imbalance; ND, not done; GBM, glioblastoma multiforme; AA, anaplastic astrocytoma.

1998; Winqvist et al., 1995), brain tumors (Sonoda et al., 1995), ovarian tumors (Viel et al., 1992), and non-small cell lung carcinomas (Tran and Newsham, 1996). In breast and lung tumors, several distinct areas of $\mathrm{LOH}$ have been identified for 11p15 (Karnik et al., 1998; Tran and Newsham, 1996), suggesting the presence of multiple tumor suppressor genes in this chromosomal region. Many of the above-mentioned tumors share $\mathrm{LOH}$ in the most distal region of this chromosomal band, centering around marker D11S1363 (Fig. 3). In addition, a second region in the more proximal part of this band has been found in breast, stomach, and lung tumors.

Our analysis of 24 normal/tumor-matched pairs of gliomas with markers encompassing chromosomal bands $11 \mathrm{p} 15.5$ and $11 \mathrm{p} 15.4$ identified allelic deletions in $33 \%$ (8 of 24) of tumors with two separate regions identified. Allelic deletion in distal Region 1 (defined by markers D11S2071 and D11S922) occurred more frequently $(75 \%)$ than in Region 2. Curiously, loss in Region 2, proximal to D11S922, appears not to be found without concomitant loss in Region 1, suggesting these more proximal events are linked mechanistically or in progression with the events in distal $11 \mathrm{p} 15.5$.

To the best of our knowledge, this work represents the first application of the novel HUGE system to LOH analyses in human tumors. The HUGE apparatus, based on a 75 - $\mu \mathrm{m}$ thin horizontal gel medium, provides the key advantage of faster run times ( 1.5 to $2 \mathrm{~h}$ ) and better resolution (sharper bands) due to minimization of thermal gradients between the bottom and top glass plates. These short runs are capable of accurately separating up to 600 base pair-length fragments, allowing ample space for the

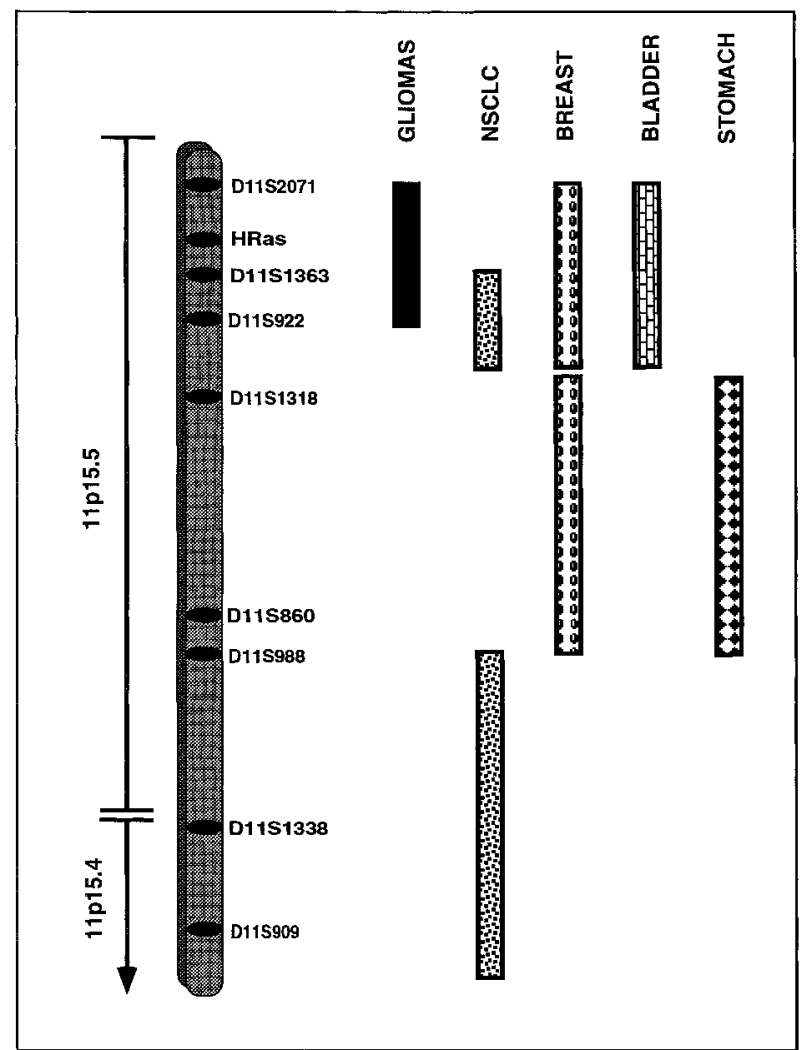

Fig. 3. Schematic representation of the regions of $\mathrm{LOH}$ determined for several types of tumors on chromosome band 11p15.5. The results of this study are depicted by the darkened bars under Gliomas. NSCLC, non-small cell lung carcinoma. 
multiplexing of markers in an $\mathrm{LOH}$ analysis. Macroscopic samples $(1$ to $2 \mu \mathrm{l})$ can be applied to this microscopic gel with ease. Our studies were additionally simplified by the direct analysis of allelic scans using Cartographer software. Peak areas are automatically calculated in each lane, eliminating the need for the separate densitometric analyses necessary in traditional ${ }^{32} \mathrm{P}$-based LOH analysis. Furthermore, the sensitivity of the laser detection method allows successful quantitative analysis of markers that occasionally amplify inefficiently in DNA samples. The combination of the novel GTI-9600 HUGE technology with the well-established $\mathrm{LOH}$ analysis reveals this to be a powerful and efficient means of detecting allelic deletions in tumors. Our study has redefined an $11 \mathrm{p} 15.5 \mathrm{LOH}$ region to $3 \mathrm{cM}$ between D11S2071 and D11S922. Such definition should allow this putative glioma tumor suppressor gene to be identified using modern genomic technologies.

\section{References}

Baffa, R., Negrini, M., Mandes, B., Rugge, M., Ranzini, G.N., Hirohaski, S., and Croce, C.M. (1996) Loss of heterozygosity for chromosome 11 in adenocarcinoma of the stomach. Cancer Res. 56, 268-272.

Bello, M.J., Vaquero, J., de Campos, J.M., Kusak, M.E., Sarasa, J.L., Saez-Castresana, J., Pestana, A., and Rey, J.A. (1994) Molecular analysis of chromosome 1 abnormalities in human glioma reveals frequent loss of $1 p$ in oligodendroglial tumors. Int. J. Cancer 57, 172-175.

Bögler, O., Huang, H.-J.S., Kleihues, P., and Cavenee, W.K. (1995) The p53 gene and its role in human brain tumors. Glia 15, 308-327.

Cawkwell, L., Bell, S.M., Lewis, F.A., Dixon, M.F., Taylor, G.R., and Quirke, P. (1993) Rapid detection of allele loss in colorectal tumours using microsatellite and fluorescent DNA technology. Br. J. Cancer 67, 1262-1267.

Fults, D., Petronio, J., Noblett, B.D., and Pedone, C.A. (1992) Chromosome $11 \mathrm{p} 15$ deletions in human malignant astrocytomas and primitive neuroectodermal tumors. Genomics 14, 799-801.

Furnari, F.B., Huang, H.-J.S., and Cavenee, W.K. (1995) Genetics and malignant progression of human brain tumours. Cancer Surv. 25, 233-275.

Karnik, P., Paris, M., Williams, B.R., Casey, G., Crowe, J., and Chen, P. (1998) Two distinct tumor suppressor loci within chromosome 11p15 implicated in breast cancer progression and metastasis. Hum. Mol. Genet. 7, 895-903.

Kleihues, P., Burger, P.C., and Scheitnauer, B.W. (1993) The new WHO classification of brain tumours. Brain Pathol. 3, 255-268.

Kraus, J.A., Koopmann, J., Kaskel, P., Maintz, D., Brandner, S., Schramm, J., Louis, D.N., Wiestler, O.D., and von Deimling, A. (1995) Shared allelic losses on chromsomes $1 p$ and $19 q$ suggest a common origin of oligodendroglioma and oligoastrocytoma. J. Neuropath. Exp. Neurol. 54 91-95.

Li, J., Yen, C., Liaw, D., Podsypanina, K., Bose, S., Wang, S.I., Puc, J., Miliaresis, C., Rodgers, L., McCombie, R., Bigner, S.H., Giovanella, B.C., Ittman, M., Tycko, B., Hibshoosh, H., Wigler, M.H., and Parsons, R. (1997) PTEN, a putative protein tyrosine phosphatase gene mutated in human brain, breast, and prostate cancer. Science 275, 1943-1947.

Shaw, M., and Knowles, M. (1995) Deletion mapping of chromosome 11 in carcinoma of the bladder. Genes Chromosomes Cancer 13, 1-8.

Sonoda, Y., Lizuka, M., Yasuda, J., Makino, R., Ono, T., Kayama, T., Yoshimoto, T., and Sekiya, T. (1995) Loss of heterozygosity at 11p15 in malignant glioma. Cancer Res. 55, 2166-2168.

Steck, P.A., Pershouse, M.A., Jasser, S.A., Yung, W.K.A., Lin, H., Ligon, A.H., Langford, L.A., Baumgard, M.L., Hattier, T., Davis, T., Frye, C., Hu, R., Swedlund, B., Teng, D.H.F., and Tavtigian, S.V. (1997) Identification of a candidate tumour suppressor gene, MMAC1, at chromosome 10q23.3 that is mutated in multiple advanced cancers. Nature Genet. 15, 356-362.

Tran, Y.K., and Newsham, I.F. (1996) High density marker analysis of 11p15.5 in non-small cell lung carcinomas reveals allelic deletion of one shared and one distinct region when compared to breast carcinomas. Cancer Res. 56, 2916-2921.

Tran, Y., Benbatoul, K., Gorse, K., Rempel, S., Futreal, A., Green, M., and Newsham, I. (1998) Novel regions of allelic deletion on chromosome 18p in tumors of the lung, brain and breast. Oncogene 17, 3499-3505.

Viel, A., Giannini, F., Tumiotto, L., Sopracordevole, F., Visentin, M.C., and Boiochhi, M. (1992) Chromosomal localisation of two putative 11p oncosuppressor genes involved in human ovarian tumours. Br. J. Cancer 66, 1030-1036.

von Deimling, A., Eibl, R.H., Ohgaki, H., Louis, D.N., von Ammon, K., Petersen, I., Kleihues, P., Chung, R.Y., Wiestler, O.D., and Seizinger, B.R. (1992) p53 mutations are associated with 17p allelic loss in grade II and grade III astrocytoma. Cancer Res. 52, 2987-2990.

Winqvist, R., Hampton, G.M., Mannermaa, A., Blanco, G., Alavaikko, M., Kiviniemi, H., Taskinen, P.J., Evans, G.A., Wright, F.A., Newsham, I., and Cavenee, W. (1995) Loss of heterozygosity for chromosome 11 in primary human breast tumors is associated with poor survival after metastasis. Cancer Res. 55, 2660-2664. 